\title{
Solução Numérica de Equações Diferenciais Usando Redes Neurais
}

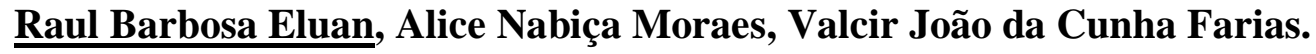 \\ Faculdade de Estatística, ICEN, UFPA. \\ 66000-000, Belém, PA
}

Email: rauleluan@gmail.com, alicenmoraes@gmail.com, valcir@ufpa.br

\section{Introdução}

Muitos problemas da Engenharia, Física e Biologia são modelados por equações diferenciais. Atualmente muitos métodos são utilizados para se obter aproximações de equações diferenciais, como por exemplo, Elementos Finitos, Diferenças Finitas, Método dos Momentos, dentre outros. Alguns desses métodos usam funções bases para representar a solução e, posteriormente, transformam o problema em um sistema linear. Outros aproximam as derivadas em alguns pontos selecionados, que constituem uma malha do domínio.

Neste trabalho são resolvidas, numericamente, equações diferenciais através de redes neurais artificiais do tipo feedforward [1,2]. Tal solução é apresentada através da soma de duas partes: a primeira é para satisfazer as condições iniciais e de contorno e a outra, é oriunda da rede neural com parâmetros ajustáveis (pesos e bias) de tal forma que minimiza uma função custo.

No treinamento da rede neural é empregado a técnica de otimização de LevenbergMaquardt. Foi considerada uma rede neural com três camadas: a camada de entrada; uma camada oculta e a camada de saída. Na camada intermediária foram adotados dois neurônios e um neurônio na camada de saída.

\section{Metodologia}

Considere que $\Psi_{t}(\vec{x}, \vec{p})$ seja a solução numérica de EDO/EDP com os parâmetros ajustáveis $\vec{p}$ (pesos e bias), o problema pode ser dado por:

$$
\min _{\vec{p}} \sum_{x_{i} \in D}\left(G\left(\vec{x}, \Psi_{t}(\vec{x}, \vec{p}), \nabla \Psi_{t}(\vec{x}, \vec{p}), \nabla^{2} \Psi_{t}(\vec{x}, \vec{p})\right)\right)^{2}
$$

Onde $G\left(\vec{x}, \Psi(\vec{x}), \nabla \Psi(\vec{x}), \nabla^{2} \Psi(\vec{x})\right)=0$ é uma equação diferencial na forma geral, $\vec{x}=\left(x_{1}, \cdots, x_{n}\right) \in \mathfrak{R}^{n}$ e $D \subset \mathfrak{R}^{n}$. Com $\Psi_{t}(\vec{x})=A(\vec{x})+F(\vec{x}, N(\vec{x}, \vec{p}))$ sendo $N(x, p)$ a saída da rede neural. Onde $A(\vec{x})$ satisfaz as condições iniciais e de contorno e não contém parâmetros ajustáveis; $N(\vec{x}, \vec{p})$ é a saída da rede neural e $F$ é construída para não contribuir com as condições iniciais/contorno.

\section{Resultados}

São apresentados dois exemplos de EDO, uma de $1^{\circ}$ (1) e outra de $2^{\circ}$ ordem (2), e um exemplo com EDP (3).

$$
\begin{aligned}
& \text { (1) }\left\{\begin{array}{l}
\frac{d \psi}{d x}+\psi=x+2 \\
\{\psi(0)=2 \quad 0 \leq x \leq 1
\end{array}\right. \\
& \text { (2) }\left\{\begin{array}{l}
\frac{d^{2} \psi}{d x^{2}}-\frac{\psi}{(x+1)}-6 * \frac{d \psi}{d x} * \frac{1}{(3 x+2)}=-x^{2}+2 \\
\psi(0)=0 \\
\frac{d \psi(0)}{d x}=0 \quad 0 \leq \mathrm{x} \leq 1
\end{array}\right.
\end{aligned}
$$


(3) $\left\{\begin{array}{l}\nabla^{2} \psi(x, y)=e^{-x}\left(x-2+y^{3}+6 y\right) \\ \psi(0, y)=y^{3} ; \\ \psi(1, y)=(1+y) e^{-1} ; \\ \psi(x, 0)=x e^{-x} ; \\ \psi(x, 1)=e^{-x}(x+1) ;\end{array}\right.$

A Figura 1(a) apresenta a solução do problema (1) (EDO de $1^{0}$ ordem), da qual observamos ter apresentado uma boa aproximação com um erro de apenas $3 \times 10^{-3}$. A Figura 1(b) apresenta a solução obtida para o problema (2) (EDO $2^{\circ}$ ordem) da qual observamos ter apresentado uma boa aproximação com um erro de apenas $4,5 \times 10^{-3}$.

(a)

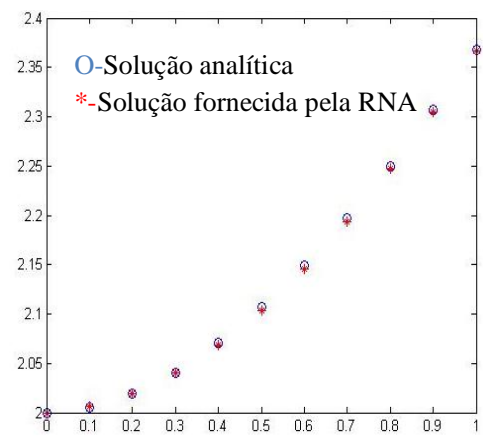

(b)

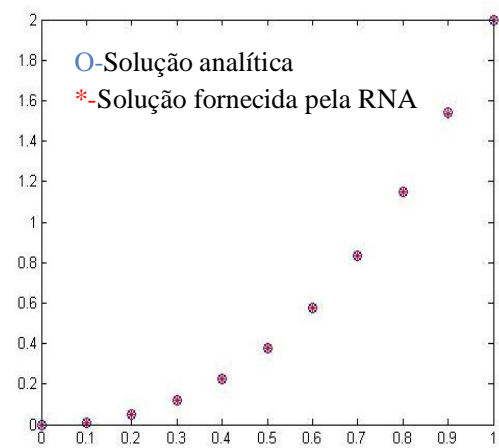

Figura 1 - (a) solução da EDO de $1^{\circ} \underline{0}$ ordem. (b) Solução da EDO de $2^{2}$ ordem.

A Figura 2 apresenta o erro da solução obtida pela rede neural multicamada com três com uma malha de $10 \times 10$ para o treinamento da rede. Como apresentou um erro máximo relativamente pequeno de $1.5 \times 10^{-3}$, então podemos concluir que a solução fornecida pela rede apresentou uma boa solução em relação à solução analítica.

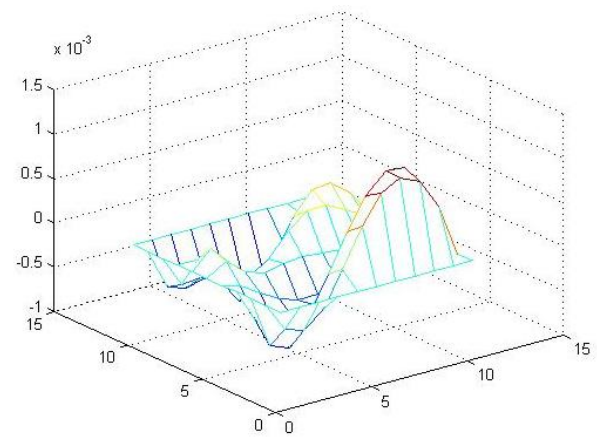

Figura 2 - Erro entre a solução fornecida pela rede e a solução analítica do problema (3).

\section{Referências}

[1] B. C. F. Batista, "Soluções de Equações Diferenciais Usando Redes Neurais de Múltiplas camadas com os métodos da Descida mais íngreme e Levenberg-Marquardt". Dissertação de mestrado, PPGME-ICEN-UFPA, 2012.

[2] I. E. L. Aristidis Likas, Artificial Neural Networks for Solving Ordinary and Partial Differential Equations. IEEE Transactions on Neural Networks, 9(1998), 987-1000.

[3] H. Lee and I. Kang, Neural algorithms for solving differential equations, J. Comput. Phys., vol. 91(1990), 110-117. 